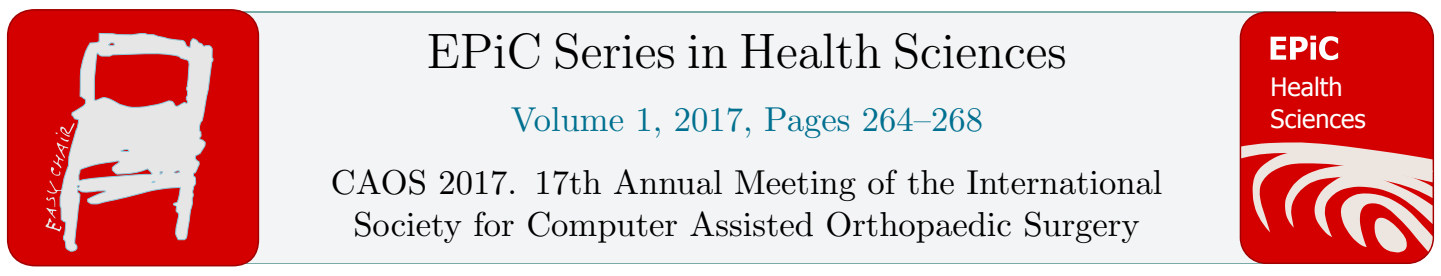

\title{
Sensor Integrated Instruments for Surgical Quality Assurance and Process Monitoring
}

\author{
Benjamin Strathen $\mathrm{MSc}^{1}$, Armin Janß $\mathrm{PhD}^{1}$, Peter Goedde $\mathrm{BSc}^{1}$ and \\ Klaus Radermacher PhD ${ }^{1}$ \\ ${ }^{1}$ Chair of Medical Engineering, Helmholtz Institute for Biomedical Engineering, RWTH Aachen \\ University, Germany
}

\begin{abstract}
Demographic changes will increase the number of surgical procedures in the next years. Therefore, quality assurance of clinical processes, such as the reprocessing of surgical instruments as well as intraoperative workflows will be of increasing importance to ensure patient safety. Surgical procedures are often complex and may involve risks for the patient. For fixation of screws, e.g. in case of pedicle screws, osteosynthesis plates or revision joint replacement surgery implants, the application of defined torques may be crucial in order to achieve optimal therapeutic results and minimal complication rates. In many cases, a subjective rating of the surgeon is necessary, as no adequate instrumentation is available. With the same subjective feeling, hammering or screwing in are performed to implant e.g. the acetabular component in total hip arthroplasty.

Our actual work is dedicated to the implementation and evaluation of functional prototypes of sensor-integrated instruments for specific types in combination with RFID technology for smart process optimization in the operating room as well as for reprocessing of surgical instruments and surgical management in combination with a knowledge-based planning, control and documentation system. Complementary (preferably wireless) sensors such for instrument identification, tracking or more complex measurements such as forces, torques, temperature or impacts during surgery as well as during reprocessing of reusable instruments could enable computer network based quality assurance in a much broader and comprehensive manner.

Within the framework of the OR.NET initiative, we follow the approach to integrate wireless sensors for measurement of temperature, force-torque as well as inertial sensors for orientation and impact control, depending on the specific type of application for monitoring of workflows during surgery as well as during reprocessing of reusable instruments and devices. The integration of smart surgical instruments into an open networked operating room based on the open communication standard IEEE 11073 knowledge-based workflow system, can help to improve the process and quality management.
\end{abstract}




\section{Introduction}

Demographic changes will increase the number of surgical procedures in the next years (Debatin et al. 2013). Therefore, quality assurance of clinical processes, such as the reprocessing of surgical instruments as well as intraoperative workflows will be of increasing importance to ensure patient safety. Furthermore, hospitals need to reduce their costs through automated processes and efficient planning of ressources (personell, infrastructure and material). Surgical procedures are often complex and may involve risks for the patient. This especially holds in case of complex procedures with multiple instrument trays and various implant sets. If problems or errors occur during the surgical procedure, therapeutical outcome and patient safety can be compromised (Wirbel et al. 2014; Saleh et al. 2015; Meeuwis et al. 2016).

For fixation of screws, e.g. in case of pedicle screws, osteosynthesis plates or revision joint replacement surgery implants, the application of defined torques may be crucial in order to achieve optimal therapeutic results and minimal complication rates. However, in many cases a subjective rating of the surgeon is necessary as no adequate instrumentation is available. Over-tightening of the screws can cause bone damage or bone interface failure, may lead to a longer healing process or implant failure (Steiner et al. 2016; Aziz, SR et al. 2014; Ab-Lazid et al. 2014; Reynolds et al. 2013). With the same subjective feeling, hammering or screwing in are performed to implant e.g. the acetabular component in THA. The introduction of surgical navigation systems established position tracking and user guidance for certain task sequences and a limited number of instruments and interventions. However, complementary (preferably wireless) sensors such for instrument identification, tracking or more complex measurements such as forces, torques, temperature or impacts during surgery as well as during reprocessing of reusable instruments could enable computer network based quality assurance in a much broader and comprehensive manner. Surgical checklists recommended by the World Health Organization (WHO) significantly reducing complication rates (Haynes et al. 2009), may be supported by computer assisted workflow monitoring on the bases of networking sensor integrated smart instruments. This contribution introduces the OR.NET concept of an integrated monitoring based on open multisensory network of smart instruments.

\section{State of the Art}

Although computer-assisted systems and sensor integrated instruments are available, they are not used in broad clinical routine. Apart from classical navigated instruments tracked by position measurement, sensor integrated instruments e.g. based on RFID technology has been proposed for surgical monitoring and workflow analysis (Boehler 2016; Glaser et al. 2015).

Moreover, application specific sensorized instruments such as e.g. the PediGuard (R) instrument for the detection of pedicle bore perforations by electrical impedance measurement (Bai et al. 2013) provide not only the information on a potential perforation as such, but could also deliver information on the actual sequence of operation. Another example is the real time recording of surgical sponges and the tracking of actions of the surgical team by using radio frequency identification (RFID) sensors (Kranzfelder et al. 2012).

\section{Approach}

Within the framework of the OR.NET initiative (www.ornet.org) we follow the approach to integrate wireless sensors for measurement of temperature, force-torque as well as inertial sensors for orientation and impact control, depending on the specific type of application for monitoring of 
workflows during surgery as well as during reprocessing of reusable instruments and devices. The integration concept is based on the open communication standard IEEE 11073 (Kasparick et al. 2015). RFID equipped instruments help to monitor automatically each step of operation and to provide related information on the central surgical workstation if needed. The option to perform bulk reading of RFID equipped instruments also enable to check completeness of instruments sets during reprocessing and packaging as well as during surgery while searching for a specific instrument in the local OR stock during an intervention. The OR.NET approach opens the door towards the Internet-ofThings (IoT) in the operating room, providing open standards for interoperability of different devices and multisensor networks for computer-assisted knowledge based workflow navigation and monitoring. Figure 1 and Figure 2 shows an exemplary panel of the OR.NET surgical workstation developed in our lab with the workflow navigation bar and integrated checklists.

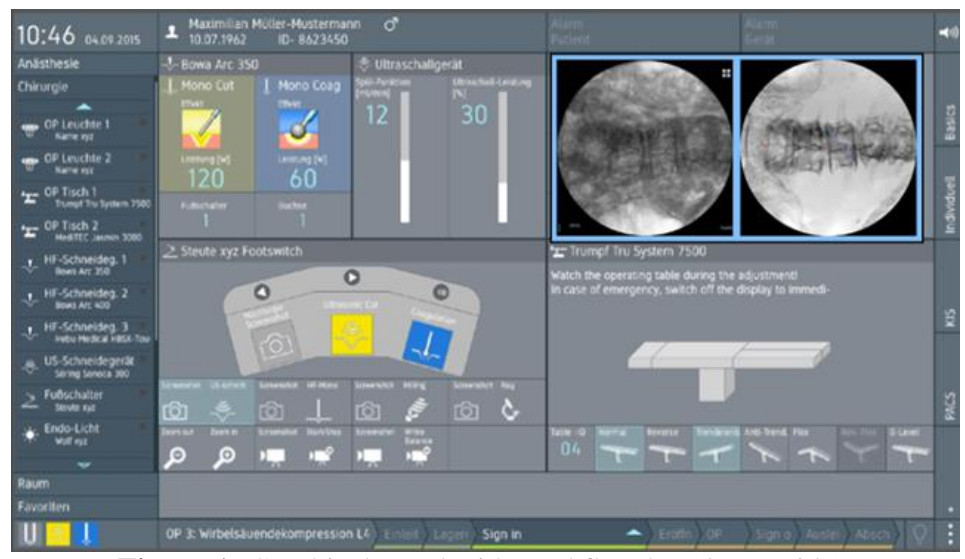

Figure 1: Graphical panel with workflow bar (lower side).

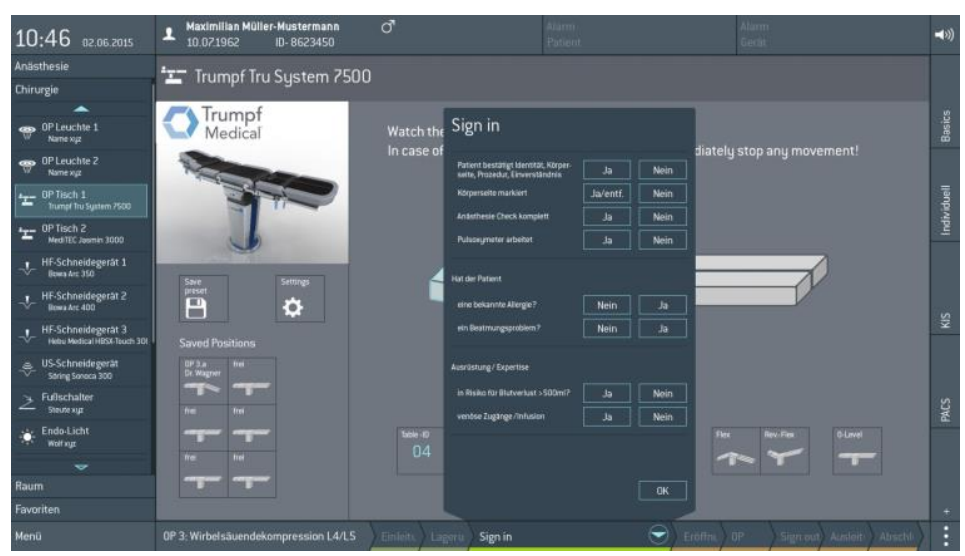

Figure 2: Surgical checklist of the OR.NET demonstrator.

The operating team or the surgeon are able to configure and document e.g. the patient information, diagnostic data, control available networked medical devices or use the workflow status from a touchbased graphical user interface (GUI) (Janß et al. 2014). 


\section{Conclusion and Outlook}

The main objective of this paper was to propose the OR.NET concept of an integrated workflow management and monitoring based on open multisensor network of smart surgical instruments. Our approach intends to support and improve quality assurance and patient safety not only during surgery but also during reprocessing, storage and handling of instruments and devices. Our actual work is dedicated to the implementation of a functional prototypes of sensor integrated instruments for specific types of intervention (especially in traumatology) and the evaluation of the sensor integrated surgical instruments in combination with RFID technology for smart process optimization in the operating room as well as for reprocessing of surgical instruments and surgical management in combination with a knowledge-based planning, control and documentation system (Andersen et al. 2015).

The integration of smart surgical instruments into an open networked operating room based on the open communication standard IEEE 11073 knowledge-based workflow system, can help to improve the process and quality management (Kasparick et al. 2015). However, in order to benefit from the integrated technology, clinics, medical device manufacturers, research institutes and lead users have to work together in order to define specific requirements as well as standard operating procedures (SOPs) and guidelines for the different surgical disciplines and procedures as a ground truth for surgical workflow management and monitoring.

\section{References}

Ab-Lazid R, Perilli E, Ryan MK, Costi JJ, Reynolds KJ, Pullout strength of cancellous screws in humanfemoral heads depends on applied insertion torque, trabecular bone microarchitecture and areal bone mineral density, JMech Behav Biomed Mater, 40, pp. 354-361, 2014.

Andersen B, Ulrich H, Rehmann D, Kock A-K, Wrage J-H, Ingenerf J, Reporting Device Observations for semantic interoperability of surgical devices and clinical information systems, Conf Proc IEEE Eng Med Biol Soc, 2015, pp. 1725-1728, 2015.

Aziz M, SR, Tsuji MR, Nicayenzi B, Crookshank MC, Bougherara H, Schemitsch EH, Zdero R, Biomechanical measurements of stopping and stripping torques during screw insertion in five types of human and artificial humeri, Proc Inst Mech Eng H, 228(5), pp. 446-455, 2014.

Bai Y-S, Niu Y-F, Chen Z-Q, Zhu X-D, Gabriel LKP, Wong HK, Li M, Comparison of the pedicle screws placement between electronic conductivity device and normal pedicle finder in posterior surgery of scoliosis, J Spinal Disord Tech, 26(6), pp. 316-320, 2013.

Boehler L, Identification of instruments and implants with RFID and Data Matrix Codes for the use at the instrument table, Electrotechnical Review, 1(11), pp. 227-230, 2016.

Debatin JF, Albat A, Krankenhausmanagement: Strategien, Konzepte, Methoden, 2., aktualisierte und erweiterte Auflage, Medizinisch Wissenschaftliche Verlagsgesellschaft, Berlin [Germany], 2013.

Glaser B, Schellenberg T, Franke S, Dänzer S, Neumuth T, Surgical instrument similarity metrics and tray analysis for multi-sensor instrument identification. In: Webster RJ, Yaniv ZR (eds.): SPIE Medical Imaging, SPIE, pp. 941526, 2015.

Haynes AB, Weiser TG, Berry WR, Lipsitz SR, Breizat A-HS, Dellinger EP, Herbosa T, Joseph S, Kibatala PL, Lapitan MCM, Merry AF, Moorthy K, Reznick RK, Taylor B, Gawande AA, A surgical safety checklist to reduce morbidity and mortality in a global population, $N$ Engl J Med, 360(5), pp. 491-499, 2009.

Janß A, Benzko J, Merz P, Dell'Anna J, Strake M, Radermacher K, Development of Medical Device UI-Profiles for Reliable and Safe Human-Machine-Interaction in the Integrated Operating Room 
of the Future. In: Proceedings of the 5th International Conference on Applied Human Factors and Ergonomics 2014, Krakow, pp. 1855-1860, 2014.

Kasparick M, Schlichting S, Golatowski F, Timmermann D, Medical DPWS: New IEEE 11073 standard for safe and interoperable medical device communication. In: 2015 IEEE Conference on Standards for Communications and Networking (CSCN), pp. 212-217.

Kranzfelder M, Zywitza D, Jell T, Schneider A, Gillen S, Friess H, Feussner H, Real-time monitoring for detection of retained surgical sponges and team motion in the surgical operation room using radio-frequency-identification (RFID) technology: a preclinical evaluation, J Surg Res, 175(2), pp. 191-198, 2012.

Meeuwis MA, Jongh MAC de, Roukema JA, van der Heijden FHWM, Verhofstad MHJ, Technical errors and complications in orthopaedic trauma surgery, Arch Orthop Trauma Surg, 136(2), pp. 185-193, 2016.

Reynolds KJ, Cleek TM, Mohtar AA, Hearn TC, Predicting cancellous bone failure during screw insertion, J Biomech, 46(6), pp. 1207-1210, 2013.

Saleh K, Ginnetti M, Curry E, Tzeng T, El-Othmani M, Patient harm and preventable error in orthopedic surgery, Int Surg J, pp. 450-456, 2015.

Steiner JA, Ferguson SJ, van Lenthe GH, Screw insertion in trabecular bone causes peri-implant bone damage, Med Eng Phys, 38(4), pp. 417-422, 2016

Wirbel R, Yacoub A, Dehne M, Prospective collection of error data in orthopaedic and trauma surgery procedures, Int Orthop, 38(11), pp. 2369-2375, 2014

\section{Acknowledgements}

This research has been funded in parts within the projects OR.NET - Secure and Dynamic Networking in Operating Room and Hospital (German Federal Ministry of Education and Research BMBF; grant no.: 16KT1203 and 16KT1238) and the project ZiMT - Certifiable Integrated Medical Technology and IT Systems based on Open Standards in the Operating Room and Hospital (State of North-Rhine Westphalia and the European Commission - European Regional Development Fund (EFRE); grant no.: EFRE-0800454). 